\title{
Application of the improved genetic algorithm in robot path planning
}

\author{
Wang Rui ${ }^{1, a}$, Wang Jinguo ${ }^{2, b^{*}}$ (corresponding author), Wang $\mathrm{Na}^{3, \mathrm{c}}$ \\ ${ }^{1}$ Department of Information Engineering, Jilin Business and Technology College \\ China \\ ${ }^{2}$ Department of Urology, the First Hospital of Jilin University, China \\ ${ }^{3}$ Department of Anaesthesiology, the First Hospital of Jilin University, China \\ aXiaoben6666@126.com, ${ }^{b}$ wangjinguolily@163.com, ${ }^{c}$ lilyly12345@163.com
}

\section{Keywords: genetic algorithm, mobile robot, path planning}

\begin{abstract}
In order to improve the algorithm efficiency, expand the scope of application, we study the mobile robot path planning technology based on improved genetic algorithm. In this paper, we design a new adaptive function, which contains the path length information, the collision penalty factor, and the path gap factor. Experimental results express that the method can search the optimal path between the starting point and the target point.
\end{abstract}

\section{Introduction}

Path planning is one of the basic problems in mobile robot motion planning. Robot path planning is a safe and efficient path for robot to accomplish a given task in its work-space. Generally speaking, there are many paths for the robot to accomplish the given task. In practice, it is often to choose a path that is optimal or approximately optimal path under certain criteria. There are some common criteria:the shortest path, the least consumption of energy or the shortest use of time, etc. Therefore, the robot path planning is essentially a constrained optimization problem[1].

Researchers have found many ways to solve the path planning problem for mobile robots. Genetic algorithm is widely used in path optimization because of its strong global optimization ability, and some research results are obtained[2].Genetic algorithm(GA) is a new parameter optimization method, which is based on natural selection principle and population evolution mechanism, and has many features that are different from the traditional optimization method[3].

Genetic algorithm uses several operators (selection, crossover and mutation) abstracted from natural selection to carry on genetic operations for the strings of the parameters, and each string corresponds to a feasible solution. However, the path coding is still used to express the robot path with not completely method. There are many invalid paths in the solutions.

In the process of calculation,we need to judge the path and add the insertion operator, delete operator to repair the invalid path. It will increase the complexity of the algorithm. On the other hand, the individuals of invalid path participate in genetic operations, it will influence the rapid convergence of genetic algorithm. So if using 
traditional genetic algorithm to solve the path planning of mobile robot, it will have the premature convergence and the solution of path planning is unstable[4].

\section{Problem description}

The basic idea of genetic algorithm for solving path planning problem is: the path individual is expressed as a series of intermediate points in the robot's trajectory, and converted to binary series.First the path group is initialized, then carry on the genetic operation, such as choice, cross, copy, variation, etc. After several generations of evolution, stopping evolution, current optimal individual is output. The process is shown as below:

$$
\lambda=: 0
$$

Random initialization group $\mathrm{P}(0)$. Calculate the fitness of individuals in group $\mathrm{p}(0)$.

While(not meeting termination criteria) do

\{

The new population $P(\lambda+1)$ is formed by genetic algorithm of $P(\lambda)$.

Calculate the individual fitness of $P(\lambda+1), \lambda=: \lambda+1$.

\}

Individual coding. The individual represents a movement path in the work-space of a robot, and the path is expressed as a series of halfway points from the starting point to the target point. Encoding the number of identity sequence as the path number, it is have no same identification sequence in each path. Because the robot movement path is changeable, therefore, the individual's length is not determined. In computer simulation, we use the maximum possible length of the individual as the individual array dimension.

Population initialization. The initial path of the robot is generated as follows: From the starting point, it the use of environmental information and local search technology, selects a non-obstacle node randomly adjacent with the starting point as the next node, so repeatedly, until you find the end point. The robot movement from the starting point $\mathrm{S}$ and the end point $\mathrm{E}$, we connect $\mathrm{E}$ and $\mathrm{S}$ with a series of random selection, freedom, and not necessarily continuous grid serial numbers. Thus, the initial population is expressed as a formula as below.

$P=\left\{P_{1}, P_{2}, \ldots, P_{n-1}, P_{N}\right\}$

In which, $P_{i}$ is the path individual. It expresses with variable length chromosome.

Path repair mechanism. The path individual in the population $\mathrm{P}$ is valid or invalid, and the path repair mechanism is used to repair the invalid path. The judging condition and repairing method of invalid path is:

The path individual contains one or more obstacle grids. In the path to remove obstacles grid, add the nearest free grid to form a new path individual. 
The path points are outside the workspace of the robot. In the workspace of the robot, the new free grid the nearest from the node is added to form the new path individual.

The path final grid is not the target point. Lengthen the path, increase the free grid, and make the path reach the target point.

Adaptive function. First, an optimized path must be not a collided, interrupted path, and then the path is the shortest path. Therefore, the path individual adaptive function should contain path length information, collisionless information and path discontinuity information, so the adaptive function is defined as follows:

$f(p)=W_{d} d(p)+W_{p} p e(p)+W_{c} c(p)$

In which, $W_{d}, W_{p}, W_{c}$ is the weight factor; $d(p)$ is the path length function, $p e(p)$ is the collision penalty function and $c(p)$ is the path gap function, the formula is defined as follows:

$d(p)=\sum_{i=1}^{p n-1} l\left(S_{i}\right)$

In which, $I\left(S_{i}\right)$ is the distance between adjacent nodes, $p n$ is the path node number.

$$
p e(p)=\sum_{i=0}^{m_{0}} \alpha \max \left(0, r-h_{i}\right)
$$

In which, $h_{i}$ is the distance between the obstacle grid center and the path, $r$ is the radius of the obstacle, $m_{0}$ is the obstacle number of the robot and obstacle collision, $\alpha$ is the penalty constant.

$$
c(p)=\sum_{i=1}^{p n-2} e^{\alpha\left(g_{i}-\tau\right)}
$$

In which, $\alpha$ is constant coefficient. The ${ }^{g_{i}}$ is the nearest distance between the $i^{\text {th }}$ path and the obstacle. $\tau$ is the ideal path to the gap distance.

\section{Experimental results and analysis}

This experiment is carried out in the $10 \times 10$ grid environment of obstacles, the initial population is 80 . The parameters are defined as follows: $W_{d}, W_{p}, W_{c}$ were 0.6 , 
$0.2,0.1 . \alpha$ is $1, r$ is $11.5 \mathrm{~cm}, h$ is $12 \mathrm{~cm}, \tau$ is 1 cell. Run the genetic algorithm 100 times, each generation end after the 300 generation. Considering the influence that the changes of terrain environment and the obstacles distribution, it must be determined that some grids may be a barrier, and then set the probability of a barrier grid. The low probability of barrier grid can generate the terrain of the tolerance, and the high probability of obstacle can produce no solution terrain. We use the improved genetic algorithm for the mobile robot path planning, the results as shown in figure 1 .

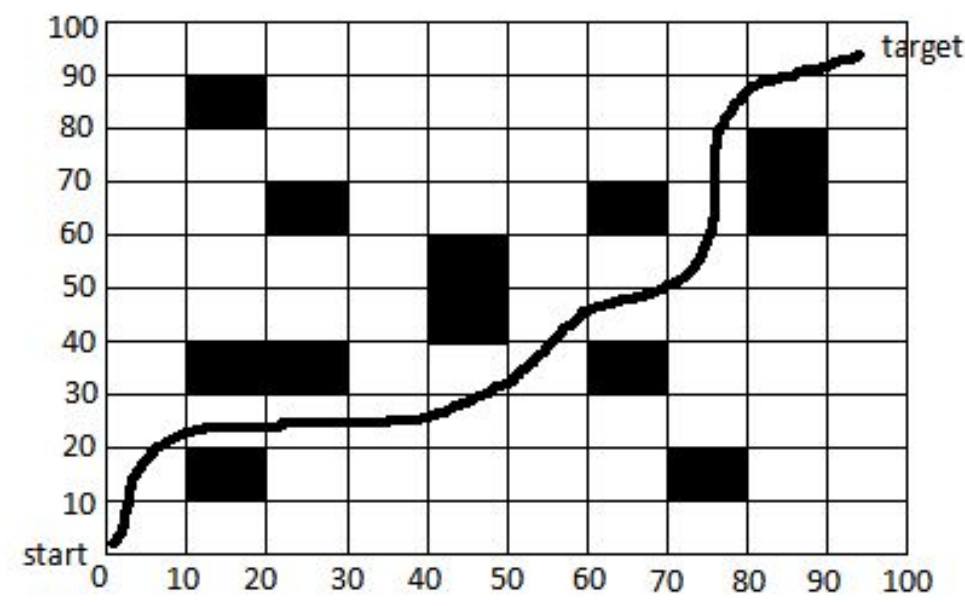

Fig. 1 the path planning of the improved GA

\section{Conclusion}

In this paper, an improved genetic algorithm is proposed. A new adaptive function is designed, which contains the path length information, the collision penalty factor, and the path gap factor. Design the path repair mechanism. The method can search the optimal path between the starting point and the target point. Experimental results and performance analysis show that the application of the improved genetic algorithm to solve the problem of robot path planning in complex environment is effective, and has strong generality.

\section{References}

[1] P. Machado,J. Tavares,F. B. Pereira,and E. Costa.Vehicle Routing Problem: Doing It the Evolutionary Way. Proceedings of the Genetic and Evolutionary Computation Conference (GECCO 2002). 2002

[2] Hongqian Liang,etc.Genetic Algorithm With Selective Generation. The Second Asia-Pacific Conference on Genetic Algorithms and Applications (APGA' 2000) . May3-52000.

[3] LIU Li,CHEN Xue-yun.Reconfiguration of distribution networksbased on fuzzy genetic algorithms. Proceedings of the ChineseSociety of Electrical Engineering . 2000 . 